\title{
Tracheobronchopathia osteochondroplastica in a patient requiring extracorporeal membrane oxygenation: a case report
}

\author{
Authors: Kritchai Vutipongsatorn, ${ }^{A}$ Eri Fujitake ${ }^{A}$ and Suveer Singh ${ }^{B}$
}

\begin{abstract}
Introduction
Tracheobronchopathia osteochondroplastica (TO) is a large airway disorder, characterised by bony nodule growth on the anterior and lateral walls of the trachea and bronchi. Its diagnosis is based on the 'rock garden' appearance on bronchoscopy. ${ }^{1-3}$ However, as an uncommon condition with an estimated incidence of $0.01-0.12 \%$, TO often goes unnoticed even though patients often have an increased risk of recurrent respiratory symptoms, such as chronic cough or haemoptysis. Here, we report a case of TO in a patient requiring extracorporeal membrane oxygenation (ECMO), which to our best knowledge has not been reported before.
\end{abstract}

\section{Methods}

A 51-year-old female presented to the local hospital with a 2-day history of dyspnoea preceded by a prodrome of myalgia, diarrhoea and fever. She was admitted and treated for an infective exacerbation of asthma with non-invasive ventilation, nebulised salbutamol and hydrocortisone, as well as tazocin and clarithromycin. Chest X-ray revealed lower lobe consolidation of both lungs (Fig 1). Subsequent deterioration led to endotracheal intubation with ventilatory support. Following hypoxaemia refractory to $100 \%$ fraction of inspired oxygen, a decision was made to transfer the patient to an intensive care unit (ITU) at a tertiary care centre on ECMO. Review by a senior respiratory intensivist identified firm interspaced pale nodules protruding from the mucosal walls within the tortuous 'corkscrew-like' airways. These extended throughout both airway trees. The computed tomography (CT) chest demonstrated prominent cartilaginous proximal airway walls (Fig 2). A diagnosis of TO was made. The patient eventually made a full recovery and was discharged back to local hospital after 48 days in ITU.

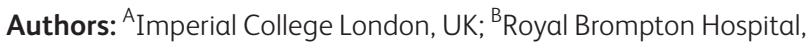
London, UK

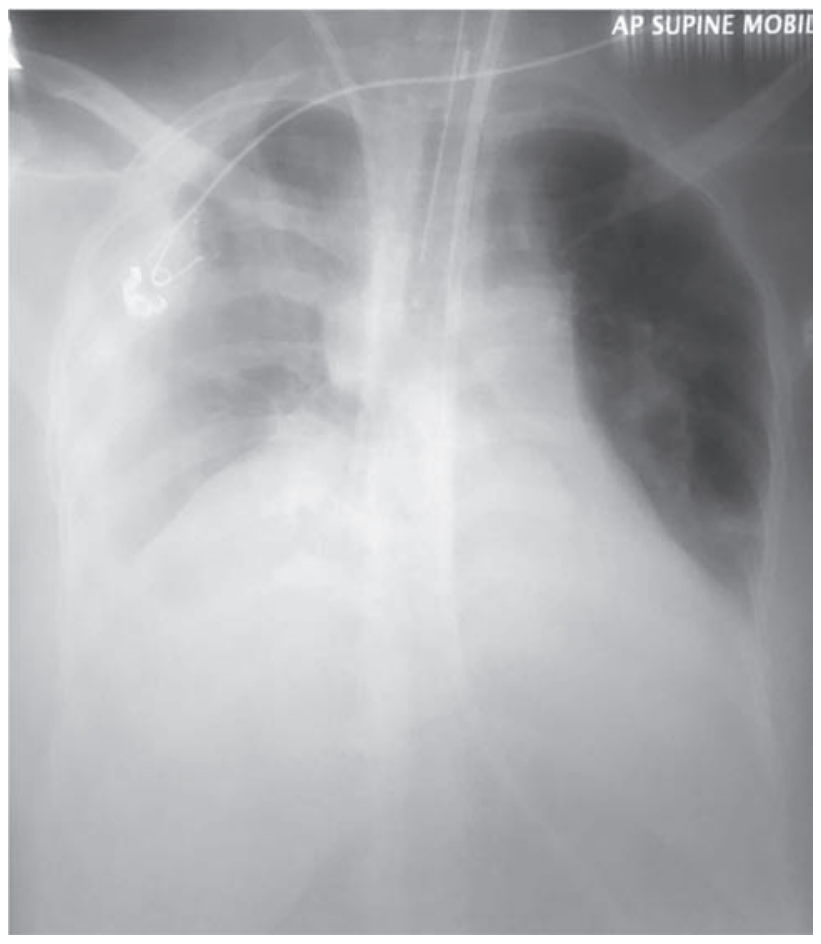

Fig 1. Chest X-ray

\section{Results and discussion}

We treated a patient with newly diagnosed TO with a primary diagnosis of meningococcal septicaemia and pneumonia requiring ECMO. In this patient, the diagnosis of TO did not change the management plan. However, we cannot rule out the possibility that TO might have contributed to the acquisition and severity of the chest infection. Furthermore, the diagnosis was only made on the sixth bronchoscopy by a specialist respiratory intensivist, highlighting the need for physicians to be familiar with the condition. 


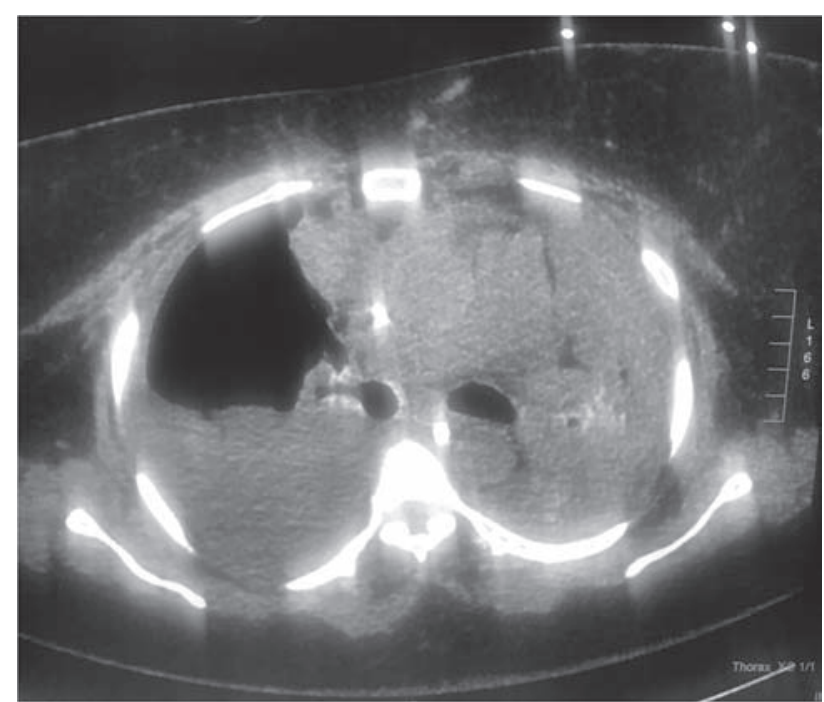

Fig 2. Computed tomography.

\section{Conclusion}

To our best knowledge, this is the first reported case of a TO patient requiring ECMO in an ITU, which demonstrates that ECMO can be effectively used in these patients.

\section{Conflict of interest statement}

None declared.

\section{References}

1 Abu-Hijleh M, Lee D, Braman SS. Tracheobronchopathia osteochondroplastica: a rare large airway disorder. Lung 2008;186:353-9.

2 Bioque JC, Feu N, Rubio JM et al. Tracheobronchopathia osteochondroplastica: clinical study and follow-up in nine cases. J Bronchology 2001;8:78-83.

3 Leske V, Lazor R, Coetmeur D et al. Tracheobronchopathia osteochondroplastica: a study of 41 patients. Medicine 2001;80:378-90. 